\title{
Calibration method for a strain-gauge measurement system based on bidirectional rail loading
}

\author{
Andrey Kolomeets ${ }^{1, *}$, and Tatyana Sych $^{1}$ \\ ${ }^{1}$ Siberian Transport University, 630049 Novosibirsk, Russia
}

\begin{abstract}
At present, all railways tend to increase the speed of passenger trains and axle loads in transportation of goods. These processes contribute to the improvement of rolling stocks and railway infrastructures. At the same time, there is a need to maintain a high level of safety in transportation. Therefore, experimental research and feasibility studies of the existing monitoring solutions are of the highest priority. Being experimental in nature, this study is aimed at increasing the reliability of the strain-gauge method in measurement of dynamic forces transferred from the wheel to the rail in real operating conditions. Rail strains were experimentally studied under bidirectional loading using a special-purpose impact device. Results of this research are presented in the paper. An experimental measuring section was created for recording loads. Strain gauges were installed on rails in several cross-sections. Rails were loaded with a special-purpose device, which was designed to adjust a loading force and shift a contact point. The structure of the loading device and its basic features are described in the paper.
\end{abstract}

\section{Introduction}

Defects of wheel tread surfaces have a negative impact on track superstructures and undercarriage of rolling stock. Growing freight traffic and increasing capacities of freight cars lead to accelerated deterioration of wheelsets and, therefore, failures of rolling stock. Systems for monitoring rolling stock in motion are developed and implemented to early detect wheelsets with wheel tread defects. Wheel tread monitoring systems are based on recording the forces transferred from wheels to rails. The strain gauge method is one of the most promising ways of recording these forces. It is based on measurements of rail strains under a passing train.

Operators and owners place high demands on operating conditions and safety of their rolling stocks. Repair stops of cars lead to delays in delivery and, therefore, economic losses. In this connection, it is important to ensure reliable measurement results and avoid rejection of defect-free wheelsets during strain-gauge monitoring.

Specialists of the Siberian Transport University (Novosibirsk) have been engaged for a long time in developing the strain-gauge method to monitor wheel tread defects in freight

* Corresponding author: andreykolomeec@yandex.ru 
cars $[3,9,13]$. In collaboration with the Chaplygin Siberian Scientific Research Institute of Aviation (SIBNIA), they improve measuring equipment, study methodological aspects of monitoring, and test the method in operation [5, 6, 14].

While moving along the rail, trains with wheel tread defects produce impact interaction in the wheel-rail system. This impact interaction is usually complex and involves both vertical and horizontal forces. Due to this, it is necessary to estimate strains of rail webs caused by simultaneous application of horizontal and vertical forces. The purpose of the paper is increase the reliability of strain-gauge monitoring aimed at detecting wheel thread defects of freight cars in motion. The scientific novelty of the study is in estimating strains of rail webs under simultaneous loading of rails with vertical and horizontal forces.

\section{Research methods}

In order to increase the reliability of monitoring results, strain-gauge sections and measuring equipment are calibrated under operating conditions. There are two types of calibration depending on predefined forces applied to the rail - static or dynamic. The calibration methods based on static forces have several modifications. Figure 1 shows the most common calibration schemes for strain-gauge sections in which static forces are used (Fig. 1a and b) and dynamic forces (Fig. 1c).

For calibration of the strain-gauge section according to the first scheme (Fig. 1a), a special-purpose expanding device is used [1]. It is fastened to the rail foot on both sides. A hydraulic or mechanical jack is placed between the upper support and the rail. Static forces are measured by an electrical dynamometer. An advantage of this calibration scheme is the mobility of the expanding device. Due to this, calibration can be conducted at any point of the measuring section. This calibration scheme does not take into account the stiffness of the rail support. The contribution of this stiffness to results of rail strain measurements can reach $10 \%[2,4]$.

It is a challenging task to calibrate the strain-gauge section, while taking into account the stiffness of the rail base in real conditions. The study [3] proposes a calibration scheme in which a hydraulic or mechanical jack is placed between a cross beam of a car and rail (Fig. 1b). This calibration procedure requires a freight car or a flat car with cargo of known weight. Strains are measured using a mechanical dynamometer with a dial gauge or an electric dynamometer. A clear advantage of this calibration procedure is the fact that the rail rests against the ballast layer and strain measurements take into account the stiffness of the rail base.

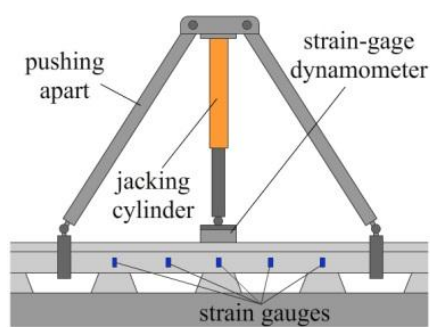

a)

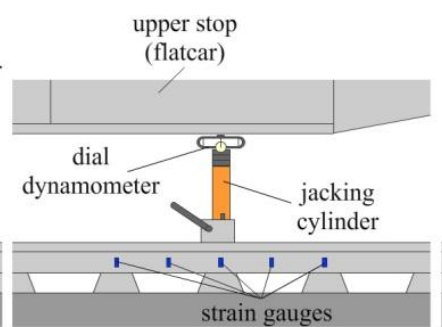

b)

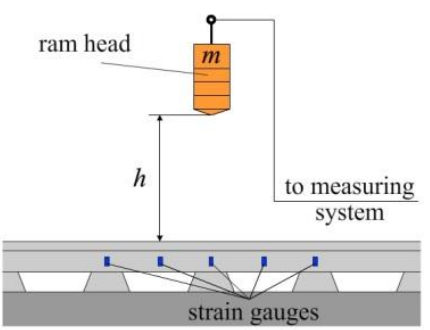

c)

Fig. 1. Calibration schemes for the strain-gauge section with: a) static force applied from the expanding device; b) static force applied to the cross beam of a loaded freight car; c) dynamic force applied from the special-purpose impact device.

Calibration of the strain-gauge section with dynamic force is subject to the third scheme (Fig. 1c). The calibration procedure is based on a special impact device of known weight, 
which is dropped onto the rail from a certain height. Timing parameters of the impact are recorded, while the force of impact is determined indirectly. An advantage of this calibration method is that it is mobile and does not require additional tools or devices. A disadvantage is that it cannot be used to identify a point of application of dynamic force (eccentricity). In case of significant eccentricity, there is a moment of force that leads to flexural (lateral) strains of rail webs.

The common drawback of the schemes described above is that they cannot be used to estimate strains of the rail from horizontal (lateral) forces. In real operating conditions, lateral forces are created by pressing a wheel flange against the rail. This study proposes a method for calibrating the strain-gauge section using a special-purpose loading device. The device applies a static force to the rail in two directions (Fig. 2).

The loading device (Fig. 2) is placed on the rail on a special holding platform 6. A horizontal (lateral) force is applied by a horizontal hydraulic cylinder 7 of model DU10P100. The force is limited to $218 \mathrm{kN}$ (22.2 tf) in the vertical direction and to $110 \mathrm{kN}$ $(11.3 \mathrm{tf})$ in the horizontal direction. The vertical hydraulic cylinder 2 (model DU20P100) rests against a cross beam of a loaded flat car from above and against a special adjustable support 6 from below. The lower support of the vertical hydraulic cylinder can be used to adjust a point of application of force by displacing it relative to the middle line of the rail head surface. The point of application of force can be changed by displacing an elastic roller installed between the rail and the support 6 . This adjustment allows vertical forces to be applied with different eccentricity.

The horizontal hydraulic cylinder 7 in the base rests against the compensation beam 8 , while the rod of the hydraulic cylinder rests against the rail head with glued strain gauges 5 . The point of impact for the horizontal hydraulic cylinder is not adjustable.

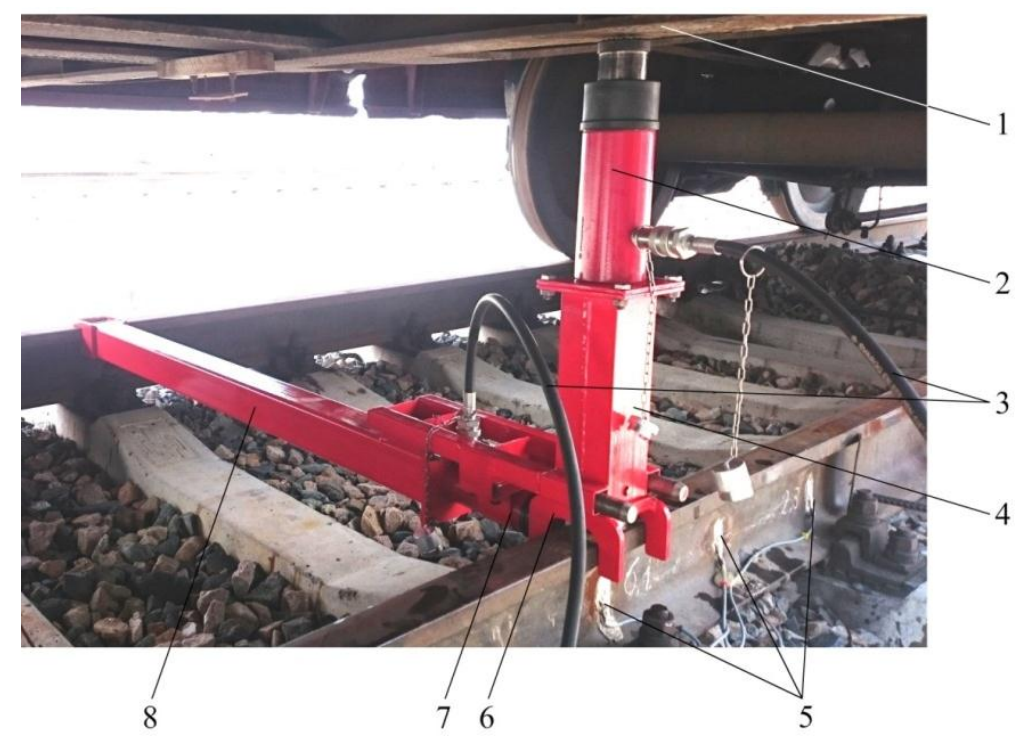

Fig. 2. Photo of the special-purpose device for bidirectional rail loading: 1 - cross beam of a loaded flat car; 2 - vertical hydraulic cylinder; 3 - high-pressure hoses; 4 - compensation support of the vertical hydraulic cylinder; 5 - strain gauges; 6 - vertical cylinder support; 7 - horizontal hydraulic cylinder; 8 - compensation beam of the horizontal cylinder.

Two independent manual hydraulic pumps NRG-7010, connected to hydraulic cylinders by high-pressure hoses 3 , are used to create pressure in hydraulic cylinders. The pressure is monitored by pressure gauges MA100VU63. The pressure gauges are installed directly on 
hand-operated hydraulic pumps. The force transferred to the rail is estimated on the basis of pressure gauge readings.

The experimental studies were conducted at a testing ground of the Siberian Transport University by using a high-speed microprocessor strain-gauge system Dynamika-3 with a sampling frequency of up to $64 \mathrm{kHz}$. The testing ground has a measuring section with wire strain gauges PKS -12-200 glued to a neutral axis of the rail web (Fig. 2, pos. 5). The strain gauges are oriented so that vertical strains can be recorded.

Rails were loaded with vertical and horizontal forces gradually and with pauses in pressure injection. Pauses in loading are necessary for recording the pressure in the system and calculating the force in order to determine a force-strain relationship.

\section{Experimental data and results}

Figure 3 presents the dependences of relative strains on time, determined experimentally by loading the rail only with lateral forces. The rail was loaded intermittently (Fig. 3a) with an increment of $18 \mathrm{kN}$. The maximum load was $54 \mathrm{kN}$, as measured by the hydraulic pressure gauge. Symmetric strains were estimated in two cross-sections. The first cross-section is located at the point of application of force. The second section is located at a distance equal to half the distance between two sleepers. In these sections, symmetric strains were equal to zero throughout the entire experiment. This suggests that the vertical force does not have any influence (Fig. 3a, pos. 1, 2). Curves 3 and 4 are based on antisymmetric strains (halfdifference) recorded during the experiment. For the cross-section in the sleeper zone, when the strain gauges were directly below the loading device, the recorded maximum strains were equal to $300 \mathrm{ppm}$ (curve 4, Fig. 3a). For the cross-section located at a distance equal to half the distance between two sleepers, the strain was decreased by three times to 100 ppm (curve 3, Fig. 3a).

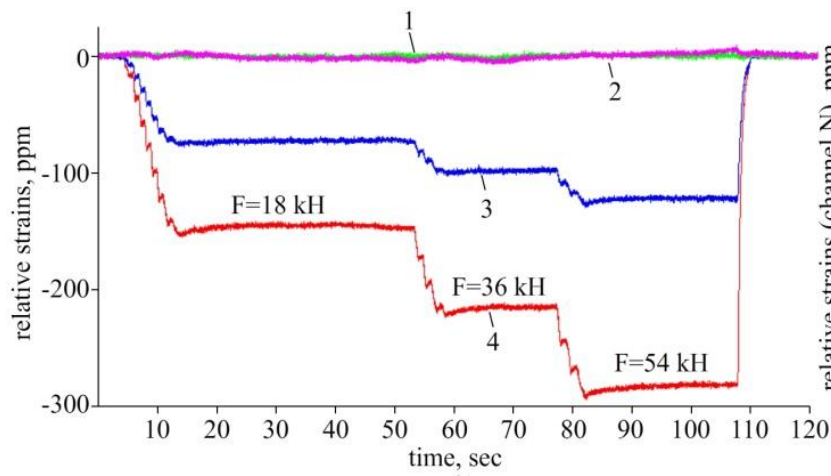

a)

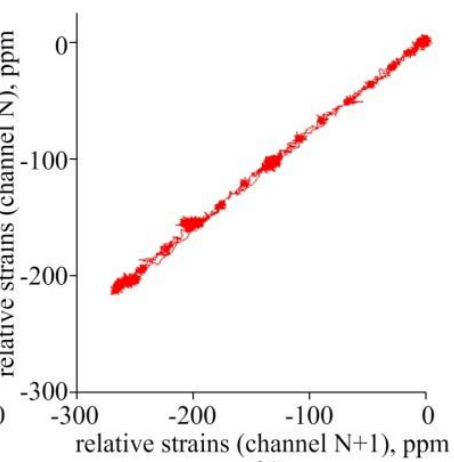

b)

Fig. 3. Graphs representing (a) dependencies of symmetric $(1,2)$ and antisymmetric $(3,4)$ strains on time; (b) correlation of readings from one pair of strain gauges when only the lateral force is applied to the rail.

The asymmetric arrangement of strain gauges in the recording system on inner and outer sides of the rail affects the reliability and accuracy of measurements [5-6]. In order to assess the effect of asymmetry, it is proposed to estimate a relationship between pairs of readings from strain gauges. A deviation of this relationship from the linear one suggests that there is a significant difference between bases of the gauges across the height of the rail. Figure $3 b$ shows a correlation graph for readings from the strain gauges located in the 
cross-section under load. The coefficient of correlation for this pair of strain gauges was 0.999, thereby suggesting that the strain gauges were positioned with a high accuracy.

In the second part of the study, only vertical forces were applied from the loading device [7-14]. Figure 4 shows the experimentally determined dependences of relative strains on time. These strains were recorded when the rail was loaded only with vertical force in a single cross-section, which was directly under the load in the sleeper zone. The rail was loaded intermittently as well. Areas with constant values of strains can be identified on the graph. These constant values were ensured by holding the rail at a certain system pressure. As in the first experiment, the increment of force was constant $-18 \mathrm{kN}$. The maximum load in the vertical direction was reached at $108 \mathrm{kN}$. The dependence of antisymmetric strains (Fig. 4a, pos. 1) is not consistent. At the first stage of the experiment, antisymmetric strains increased up to $+20 \mathrm{ppm}$. Then the distribution of strains in the rail web and the bending moment changed the sign. The strains gradually decreased up to -20 ppm. "Jumps" of values could also be observed at the end of each symmetric strain area (Fig. 4a, pos. 2), especially in the first part of the experiment until a load of $54 \mathrm{kN}$ was reached. When only vertical forces were applied, the coefficient of correlation for the values recorded by one pair of strain gauges was equal to 0.97 .

Figure $4 \mathrm{~b}$ shows a dependence of symmetric relative strains of the rail web on the applied vertical force. The dependence is linear. The deviation from the linear law estimated by the least squares method is less than $5 \%$.

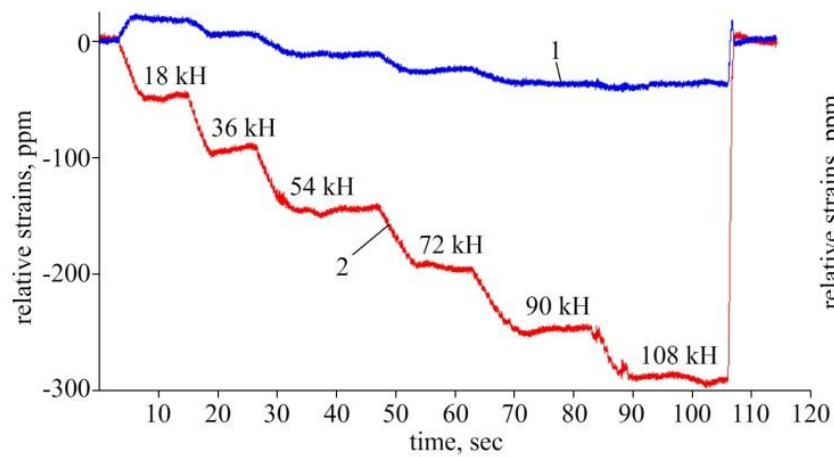

a)

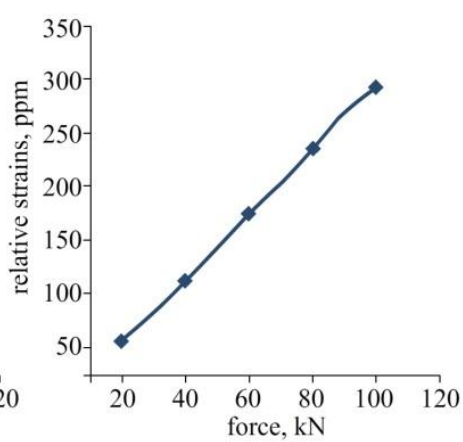

b)

Fig. 4. Dependencies of (a) antisymmetric (1) and symmetric (2) strains on time; (b) symmetric relative strains on the vertical force applied to the rail.

\section{Conclusion}

The paper considers the most common calibration schemes for strain-gauge monitoring sections in the wheel-rail system, and describes their features and limitations. The critical factors for this practical task are boundary conditions and local fastening of devices on the rail, which will determine the accuracy of calibration results. The proposed device of new design can be used for bidirectional loading of rails and adjusting the loading force. In addition, it allows a device-rail contact point to be fixed and adjusted. The calibration procedure takes into account the stiffness of the rail base.

Dependences of symmetric and antisymmetric strains on lateral and vertical forces were determined. When a lateral force is applied, the effect of symmetric strains is minimal (within the margin of error) and can be judged from antisymmetric strains. When a vertical force is applied, the dependence of symmetric strains of the rail web on the recorded force is linear throughout the range, with an accuracy of 0.95 . This level is sufficient to calibrate the monitoring section. In this case, the effect of antisymmetric flexural strains caused by 
rail canting and eccentricity of the applied vertical force (which is usual for any practical task) does not exceed $6.9 \%$.

In the future, it is planned to conduct experiments at different stiffness values of the rail base. In order to assess the effect of asymmetric positioning of strain gauges on measurement of symmetric and antisymmetric strains, a series of experiments will be conducted on a specially prepared measuring section. Strain gauges will be glued to rails with a displacement relative to their neutral lines.

\section{References}

1. Wheel Condition Monitor (WCM) / Trackside Intelligence Pty Ltd (TracklQ) (King William St, Kent Town, 2007).

2. K. Sekuła, P. Kołakowski, Proceedings of ISMA 2010 including US201 (2013).

3. S.A. Bekher, A.O. Kolomeets, Journal of Physics: Conference Series, 671 (2016).

4. Precise Diagnosis of Wheel Defects and Vehicle Weights, Signal and System Technik, 8 (2012).

5. S.A. Bekher, L.N. Stepanova, A.O. Kolomeets, A.A. Popkov, RU Patent 2625256 (2016). (in Russian)

6. L.N. Stepanova, S.I. Kabanov, E.Yu. Lebedev et al., RU Patent 2499237 (2013). (in Russian)

7. GOST P 52728-2007. Method of strain-and-temperature measurements in situ. General requirements (Standartinform, Moscow, 2007). (in Russian)

8. GOST P 55050-2012. Railway rolling stock. Permissible exposure norms to the railway track and test methods (Standartinform, Moscow, 2013). (in Russian)

9. S.A. Bekher, A.S. Kochetkov, The Siberian Transport University Bulletin, 17 (2007). (in Russian)

10. A.N. Baybakov, K.I. Kuchinskiy, S.V. Plotnikov et al., Modern problems of science and education, 6 (2013). (in Russian)

11. K.V. Eliseev, Nauka i Obrazovanie [Science and Education], 4 (2014). (in Russian)

12. A.Ya. Kogan, Dinamika puti i ego vzaimodejstvie s podvizhnym sostavom [The dynamics of the track and its interaction with the rolling stock] (Transport, Moscow, 1997). (in Russian)

13. A.S. Kochetkov, Thesis of Candidate of technical sciences, Tomsk Polytechnic University, Tomsk, 2011. (in Russian)

14. S.A. Bekher, L.N. Stepanova, A.S. Kochetkov, RU Patent 2480711 (2013). (in Russian) 